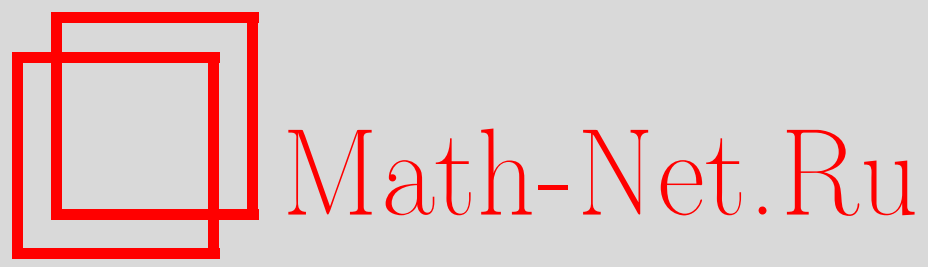

А. Г. Александров, Кратный вычет и весовая фильтрация на логарифмическом комплексе де Рама, Функи. анализ и его прил., 2013, том 47, выпуск 4, 1-17

DOI: https://doi.org/10.4213/faa3126

Использование Общероссийского математического портала MathNet.Ru подразумевает, что вы прочитали и согласны с пользовательским соглашением

http://www . mathnet.ru/rus/agreement

Параметры загрузки:

IP : 35.173 .219 .12

26 апреля 2023 г., 05:16:17

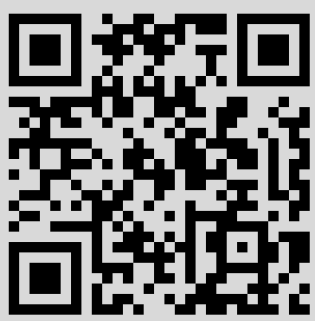


Функционалъный анализ и его приложения

2013, т. 47, вып. 4, с. 1-17

УДК 515.17

\title{
Кратный вычет и весовая фильтрация на логарифмическом комплексе де Рама
}

\author{
(C) 2013. А. Г. АЛЕКСАНДРОВ
}

\begin{abstract}
В статье изучается кратный вычет логарифмических дифференциальных форм с полюсами на приводимом дивизоре, вычислены ядро и образ отображения кратного вычета. В качестве одного из приложений получено описание весовой фильтрации на логарифмическом комплексе де Рама для дивизоров, неприводимые компоненты которых локально задаются регулярной последовательностью голоморфных функций. В частности, это позволяет вычислять смешанную структуру Ходжа на когомологиях дополнения для некоторых типов дивизоров без использования теорем о разрешении особенностей и стандартной редукции к случаю нормальных пересечений.
\end{abstract}

\section{Введение}

Понятие весовой фильтрации на логарифмическом комплексе де Рама для дивизоров с нормальными пересечениями на неособом многообразии было введено Делинем [6] в 1972 г. при описании смешанной структуры Ходжа (CCX) на когомологиях дополнения дивизора. С той поры эта теория интенсивно развивается во многих направлениях для самых разных типов многообразий и теорий когомологий, при этом практически все известные варианты обобщений основаны на редукции рассматриваемой ситуации к случаю дивизора с нормальными пересечениями, использованию общих теорем о разрешении особенностей и функториальности понятия ССХ.

В настоящей статье изучается кратный вычет логарифмических дифференциальных форм и с его помощью получено естественное описание весовой фильтрации на логарифмическом комплексе де Рама для дивизоров, неприводимые компоненты которых локально задаются регулярной последовательностью голоморфных функций. В частности, это позволяет вычислить ССХ на когомологиях дополнения для некоторых типов дивизоров, не прибегая к упомянутой выше редукции.

В первых двух параграфах рассматриваются некоторые свойства логарифмических дифференциальных форм с полюсами вдоль приводимого дивизора. В $\S \S 3$ и 4 обсуждается понятие кратного вычета относительно полного пересечения для мероморфных дифференциальных форм с простыми полюсами вдоль дивизора. В следующих двух параграфах вычисляются ядро и образ отображения кратного вычета для логарифмических дифференциальных форм. В заключительном $\S 7$ полученные результаты применяются для описания весовой фильтрации на логарифмическом комплексе де Рама и обсуждаются некоторые приложения.

\section{§1. Логарифмический комплекс де Рама}

Пусть $X$ - комплексное аналитическое многообразие размерности $m \geqslant 1$, $\Omega_{X}^{q}, q \geqslant 0,-$ пучки ростков голоморфных дифференциальных $q$-форм на 
$X$ и $D \subset X-$ (локально главный) дивизор Картье. В окрестности любой точки $x \in D$ росток $(D, x)$ задается голоморфной функцией $h \in \mathscr{O}_{X, x}$ : если $z=\left(z_{1}, \ldots, z_{m}\right)$ - локальная система координат в окрестности этой точки, то $h(z)=0$ - локальное уравнение дивизора $D$ в $x$, так что $\mathscr{O}_{D, x} \cong \mathscr{O}_{X, x} /(h)$.

Слои когерентного аналитического пучка логарифмических дифференииальных борм $\Omega_{X}^{q}(\log D), q \geqslant 0$, состоят из ростков мероморфных $q$-форм на $X$ с полюсами вдоль $D$, таких, что $h \omega$ и $h d \omega$ голоморфны в точке $x$, т. е. $h \cdot \Omega_{X, x}^{q}(\log D) \subseteq \Omega_{X, x}^{q}$ и $h \wedge d \Omega_{X, x}^{q}(\log D) \subseteq \Omega_{X, x}^{q+1}$. Ясно, что второе условие эквивалентно включению $d h \wedge \Omega_{X, x}^{q}(\log D) \subseteq \Omega_{X, x}^{q+1}$.

По определению $\Omega_{X}^{0}(\log D) \cong \mathscr{O}_{X}$ и существуют естественные вложения $\Omega_{X}^{q} \subseteq \Omega_{X}^{q}(\log D)$, которые для всех $x \notin D$ индуцируют изоморфизмы $\Omega_{X, x}^{q} \cong$ $\Omega_{X, x}^{q}(\log D), q \geqslant 0$. Кроме того, $\Omega_{X, x}^{m}(\log D) \cong \mathscr{O}_{X, x}\left(d z_{1} \wedge \cdots \wedge d z_{m} / h\right)$.

Семейство $\mathscr{O}_{X}$-модулей $\Omega_{X}^{q}(\log D), q \geqslant 0$, снабженное дифференциалом $d$, индуцированным дифференцированием де Рама, заданным на $\Omega_{X}^{\bullet}$, образует возрастающий комплекс, который называется логарифмическим комплексом де Рама. Далее, пучки логарифмических дифференциальных форм - это $\mathscr{O}_{X}$-модули конечного типа, а их прямая сумма $\bigoplus_{q=0}^{m} \Omega_{X}^{q}(\log D)$ образует внешнюю $\mathscr{O}_{X}$-алгебру, замкнутую относительно действия $d$.

\section{§2. Логарифмические формы с полюсами на приводимом дивизоре}

Для простоты будем далее предполагать, что дивизор $D \subset X$ приведен, т. е. кратности всех его неприводимых компонент равны единице. Тогда в окрестности любой точки $x \in D$ локальное уравнение дивизора $D$ задается ростком голоморфной функции $h \in \mathscr{O}_{X, x}$ без кратных множителей.

Рассмотрим несколько простых свойств логарифмических дифференциальных форм с полюсами на $D$, которые будут использоваться в дальнейшем. Сначала напомним, что $\mathscr{O}_{X}$-модуль векторных полей, логарифмических вдоль дивизора $D \subset X$, состоит из ростков голоморфных векторных полей $\mathscr{V} \in \operatorname{Der}\left(\mathscr{O}_{X}\right)$ на $X$, таких, что $\mathscr{V}(h)$ принадлежит идеалу $(h) \cdot \mathscr{O}_{X}$. В частности, $\mathscr{V}$ касается дивизора $D$ в его неособых точках. Этот модуль обозначается через $\operatorname{Der}_{X}(\log D)$. Далее, стягивание дифференциальных форм вдоль векторных полей индуцирует совершенное спаривание (см. [13]):

$$
\operatorname{Der}_{X}(\log D) \times \Omega_{X}^{1}(\log D) \rightarrow \mathscr{O}_{X} .
$$

Утверждение 1. Предположим, что $D_{i}$ - это компоненты несократимого (не обязательно неприводимого) разложения дивизора $D$, заданные функииями $h_{i}, i=1, \ldots, k$, maк что $D=D_{1} \cup \cdots \cup D_{k}$. Тогда

$$
\operatorname{Der}_{X}\left(\log D_{1}\right) \cap \cdots \cap \operatorname{Der}_{X}\left(\log D_{k}\right)=\operatorname{Der}_{X}(\log D) .
$$

Доказательство. Проверим это свойство локально. Очевидно, что левая часть содержится в правой. Обратно, пусть $\mathscr{V} \in \operatorname{Der}_{X, x}(\log D)$. Тогда

$$
\mathscr{V}(h)=\sum_{i=1}^{k}\left(h_{1} \cdots \widehat{h_{i}} \cdots h_{k}\right) \mathscr{V}\left(h_{i}\right)=f h,
$$

где $f \in \mathscr{O}_{X, x}$. После деления на $h_{i}$ обеих частей второго равенства получаем, что функция $\left(h_{1} \cdots \widehat{h_{i}} \cdots h_{k}\right) \mathscr{V}\left(h_{i}\right) / h_{i}$ голоморфна, т. е. $h_{i}$ делит $\mathscr{V}\left(h_{i}\right)$. Следовательно, $\mathscr{V}\left(h_{i}\right) \in\left(h_{i}\right) \mathscr{O}_{X, x}$ для всех $i=1, \ldots, k$. 
Следующее утверждение можно рассматривать как один из возможных дуальных вариантов утверждения 1 относительно спаривания (1).

Утверждение 2. При тех же условиях предположим, что $\Omega_{X}^{1}(\log D)$ локально порождается замкнутыми формами. Тогда существует изоморфизм

$$
\Omega_{X}^{1}\left(\log D_{1}\right)+\cdots+\Omega_{X}^{1}\left(\log D_{k}\right) \cong \Omega_{X}^{1}(\log D) .
$$

Доказательство. Ввиду теоремы 2.9 из работы [13] условие замкнутости образующих модуля $\Omega_{X, x}^{1}(\log D)$ эквивалентно изоморфизму

$$
\sum_{i=1}^{k} \mathscr{O}_{X, x} \frac{d h_{i}}{h_{i}}+\Omega_{X, x}^{1} \cong \Omega_{X, x}^{1}(\log D) .
$$

С другой стороны, $\frac{d h_{i}}{h_{i}} \in \Omega_{X, x}^{1}\left(\log D_{i}\right)$ и $\sum_{i=1}^{k} \Omega_{X}^{1}\left(\log D_{i}\right) \hookrightarrow \Omega_{X}^{1}(\log D)-$ естественное вложение. Отсюда следует утверждение.

Предложение 1. Пусть $\widehat{D_{i}}=D_{1} \cup \cdots \cup D_{i-1} \cup D_{i+1} \cup \cdots \cup D_{k}, i=1, \ldots, k$. Тогда в условиях предњдущих утверждений существуют естественные вложения

$h_{i} \Omega_{X, x}^{\bullet}(\log D) \subseteq \Omega_{X, x}^{\bullet}\left(\log \widehat{D_{i}}\right), \quad d h_{i} \wedge \Omega_{X, x}^{\bullet}(\log D) \subseteq \Omega_{X, x}^{\bullet+1}\left(\log \widehat{D_{i}}\right), \quad i=1, \ldots, k$.

Другими словами, внешнее умножение на форму $d h_{i}$, равно как и умножение на функиию $h_{i}$, «гасит» полюсы логарифмической бормы $\omega \in \Omega_{X, x}^{\bullet}(\log D)$, расположснные на компоненте $D_{i}$.

Доказательство. Рассмотрим случай $k=2$. Пусть $i=1$ и $x \in D_{1} \cap D_{2}$. Покажем, что имеет место первое включение, т. е. $h_{2} \Omega_{X, x}^{\bullet}(\log D) \subseteq \Omega_{X, x}^{\bullet}\left(\log D_{1}\right)$. По условию $h_{1}\left(h_{2} \omega\right)=h \omega \in \Omega_{X, x}^{\bullet}$. Далее,

$$
d h \wedge\left(h_{2} \omega\right)=h_{2} d h_{1} \wedge\left(h_{2} \omega\right)+h_{1} d h_{2} \wedge\left(h_{2} \omega\right)=h_{2} d h_{1} \wedge\left(h_{2} \omega\right)+d h_{2} \wedge(h \omega) .
$$

Так как дифференциальная форма $d h \wedge \omega \in \Omega_{X, x}^{\bullet}$ голоморфна, то $d h \wedge\left(h_{2} \omega\right)$ - также голоморфная форма. Аналогично, $d h_{2} \wedge(h \omega) \in \Omega_{X, x}^{\bullet}$. Следовательно, $h_{2} d h_{1} \wedge\left(h_{2} \omega\right)=h_{2}^{2} d h_{1} \wedge \omega \in \Omega_{X, x}^{\bullet}$. Пусть $d h_{1} \wedge \omega=\vartheta / h_{2}^{2}$, где $\vartheta \in \Omega_{X, x}^{\bullet}$. Теперь заметим, что $\frac{d h_{1}}{h_{1}} \in \Omega_{X, x}^{\bullet}(\log D)$, так что $\frac{d h_{1}}{h_{1}} \wedge \omega \in \Omega_{X, x}^{\bullet}(\log D)$ ввиду $\wedge$-замкнутости. Поэтому, $\frac{d h_{1}}{h_{1}} \wedge \omega=\frac{\vartheta}{h_{1} h_{2}^{2}} \in \Omega_{X, x}^{\bullet}(\log D)$, т. е. $\frac{\vartheta}{h_{2}} \in(h) \Omega_{X, x}^{\bullet}(\log D) \subseteq \Omega_{X, x}^{\bullet}$. Следовательно, $\vartheta \in\left(h_{2}\right) \Omega_{X, x}^{\bullet}$, или $d h_{1} \wedge \omega=\vartheta^{\prime} / h_{2}$, где $\vartheta^{\prime} \in \Omega_{X, x}^{\bullet}$. Таким образом, $h_{2}\left(d h_{1} \wedge \omega\right)=d h_{1} \wedge\left(h_{2} \omega\right) \in \Omega_{X, x}^{\bullet}$, т. е. $d h_{1} \wedge\left(h_{2} \omega\right)$ - голоморфная форма. Значит, $h_{2} \omega \in \Omega_{X, x}^{\bullet}\left(\log D_{1}\right)$, что и требовалось доказать.

Существование второго включения доказывается аналогично. Действительно, дифференциальная форма $h_{1}\left(d h_{2} \wedge \omega\right) \in \Omega_{X, x}^{\bullet}$ голоморфна, поскольку она представляется в виде разности $d h \wedge \omega-h_{2} d h_{1} \wedge \omega$, в которой первая форма голоморфна по условию, а голоморфность второй установлена при доказательстве первого включения. Далее,

$$
d h_{1} \wedge\left(d h_{2} \wedge \omega\right)=d\left(h_{1} d h_{2} \wedge \omega\right)+h_{1}\left(d h_{2} \wedge d \omega\right) .
$$

Так как форма $h_{1} d h_{2} \wedge \omega$ голоморфна, то и ее дифференциал также голоморфен. Наконец,

$$
h_{1}\left(d h_{2} \wedge d \omega\right)=d h \wedge d \omega-h_{2}\left(d h_{1} \wedge d \omega\right) .
$$


Форма $d h \wedge d \omega$ голоморфна по предположению, так как внешняя алгебра $\Omega_{X, x}^{\bullet}(\log D)$ замкнута относительно дифференцирования де Рама $d$, а потому $d \omega \in \Omega_{X, x}^{\bullet}(\log D)$. Как и при доказательстве первого включения, получаем, что $h_{2}\left(d h_{1} \wedge d \omega\right)$ - голоморфная форма. Отсюда следует, что $h_{1}\left(d h_{2} \wedge d \omega\right)$, равно как и $h_{1}\left(d h_{2} \wedge \omega\right),-$ голоморфные формы. Таким образом, $d h_{2} \wedge \omega \in \Omega_{X, x}^{\bullet}\left(\log D_{1}\right)$, что и требуется. Общий случай $k>2$ разбирается аналогично.

Замечание 1. Такими же рассуждениями можно показать, что для всех $i=1, \ldots, k$ существуют вложения

$$
\begin{gathered}
\left(h_{1} \cdots \widehat{h_{i}} \cdots h_{k}\right) \Omega_{X, x}^{\bullet}(\log D) \subseteq \Omega_{X, x}^{\bullet}\left(\log D_{i}\right), \\
d\left(h_{1} \cdots \widehat{h}_{i} \cdots h_{k}\right) \wedge \Omega_{X, x}^{\bullet-1}(\log D) \subseteq \Omega_{X, x}^{\bullet}\left(\log D_{i}\right) .
\end{gathered}
$$

Аналогичные включения имеются для дивизоров, составленных из произвольных наборов различных компонент (без повторений) несократимого представления дивизора $D$.

Утверждение 3. Предположим, что компоненты несократимого разложения приведенного дивизора $D$ локально задаются регулярной $\mathscr{O}_{X, x}$-последовательностъю функиий $h_{i}, i=1, \ldots, k$. Тогда

$$
\Omega_{X}^{\bullet}\left(\log \widehat{D_{1}}\right) \cap \cdots \cap \Omega_{X}^{\bullet}\left(\log \widehat{D_{k}}\right)=\Omega_{X}^{\bullet}
$$

и существует точная последовательность комплексов

$$
0 \longrightarrow \Omega_{X}^{\bullet} \longrightarrow \bigoplus \Omega_{X}^{\bullet}\left(\log \widehat{D_{i}}\right) \longrightarrow \sum \Omega_{X}^{\bullet}\left(\log \widehat{D_{i}}\right) \longrightarrow 0
$$

Доказательство. Достаточно установить справедливость равенства локально. Ясно, что правая часть содержится в левой. Рассмотрим дифференциальную $q$-форму $\omega$ из левой части. Тогда $\left(h_{1} \cdots \widehat{h_{i}} \cdots h_{k}\right) \omega \in \Omega_{X, x}^{q}$ для любого $i=1, \ldots, k$. Следовательно, $\omega \in \bigcap \frac{1}{\left(h_{1} \cdots \hat{h}_{i} \cdots h_{k}\right)} \Omega_{X, x}^{q}$, что эквивалентно включению $h \omega \in\left(h_{1}\right) \Omega_{X, x}^{q} \cap \cdots \cap\left(h_{k}\right) \Omega_{X, x}^{q}$. Из простейших свойств регулярных последовательностей вытекает, что при сделанных предположениях пересечение главных идеалов совпадает с их произведением $\left(h_{1} \cdots h_{k}\right) \Omega_{X, x}^{q}$. Отсюда следует, что $\omega \in \Omega_{X, x}^{q}$, т. е. форма $\omega$ голоморфна.

\section{§3. Разложение мероморфных форм вдоль полного пересечения}

Пусть $D=D_{1} \cup \cdots \cup D_{k}-$ приведенный приводимый дивизор. Обозначим $\mathscr{O}_{X}$-модули мероморфных дифференциальных форм степени $q \geqslant 1$, образованные дифференциальными $q$-формами с простыми полюсами и с полюсами произвольного порядка на дивизоре $\widehat{D_{j}}=D_{1} \cup \cdots \cup D_{j-1} \cup D_{j+1} \cup \cdots \cup D_{k}$, через $\Omega_{X}^{q}\left(\widehat{D_{j}}\right)$ и $\Omega_{X}^{q}\left(\star \widehat{D_{j}}\right), j=1, \ldots, k$, соответственно. Будем считать, что $\widehat{D_{1}}=\varnothing$ при $k=1$, так что $\Omega_{X}^{q}\left(\widehat{D_{1}}\right)=\Omega_{X}^{q}\left(\star \widehat{D_{1}}\right)=\Omega_{X}^{q}$.

Предположим, что комплексное аналитическое пространство $C=D_{1} \cap \cdots \cap D_{k}$ - полное пересечение. Это означает, что идеал $\mathscr{I}$, задающий $C$ в некоторой окрестности $U$ отмеченной точки, локально порождается регулярной $\mathscr{O}_{U}$-последовательностью $\left(h_{1}, \ldots, h_{k}\right)$ и $\operatorname{dim} C=m-k \geqslant 0$. Будем также предполагать, что пространство $C=C_{\text {red }}$ приведено, другими словами, $\mathscr{I}=\sqrt{\mathscr{I}}-p a \partial u$ кальный идеал. В частности, из этих условий следует, что дифференциальная 
форма $d h_{1} \wedge \cdots \wedge d h_{k}$ степени $k$ не обращается тождественно в нуль ни на одной неприводимой компоненте ростка пространства $C$. Следующее утверждение вместе с доказательством - это несколько измененная версия соответствующих рассуждений из работ [2], [3].

Теорема 1. Пусть функиии $h_{i} \in \mathscr{O}_{U}, i=1, \ldots, k$, задают локальные уравнения дивизоров $D_{i}$ в окрестности $U$ точки $x \in C$. Предположим, что мероморфная дифференииальная форма $\omega \in \Omega_{U}^{q}(D)$ удовлетворяет условиям

$$
d h_{j} \wedge \omega \in \sum_{i=1}^{k} \Omega_{U}^{q+1}\left(\widehat{D_{i}}\right), \quad j=1, \ldots, k .
$$

Тогда существует голоморфная функиия $g$, которая не обращается тождественно в нуль ни на одной неприводимой компоненте полного пересечения $C$, голоморфная дифберенииальная борма $\xi \in \Omega_{U}^{q-k}$ и мероморфная q-форма $\eta \in$ $\sum_{i=1}^{k} \Omega_{U}^{q}\left(\widehat{D_{i}}\right)$, такuе, чmо

$$
g \omega=\frac{d h_{1}}{h_{1}} \wedge \cdots \wedge \frac{d h_{k}}{h_{k}} \wedge \xi+\eta .
$$

Доказательство. В окрестности точки $x \in U$ дифференциальную форму $\omega$ можно записать в виде

$$
\omega=\frac{1}{h_{1} \cdots h_{k}} \sum_{|I|=q} a_{I}(z) \cdot d z_{I}
$$

где $I:=I^{q}=\left(i_{1}, \ldots, i_{q}\right), 1 \leqslant i_{1}, \ldots, i_{q} \leqslant m$, - мульти-индекс, $d z_{I}=d z_{i_{1}} \wedge \cdots \wedge d z_{i_{q}}$ и $a_{I}(z) \in \mathscr{O}_{U}-$ множество коэффициентов, кососимметрических относительно $I$. Очевидно, что условия (2) эквивалентны включениям

$$
d h_{j} \wedge \sum_{I} a_{I}(z) \cdot d z_{I} \in \sum_{\ell=1}^{k} h_{\ell} \Omega_{U}^{q+1}, \quad j=1, \ldots, k,
$$

которые приводят к следующей системе соотношений между коэффициентами $a_{I}$ и частными производными функций $h_{j}$ :

$$
\sum_{\ell=1}^{q}(-1)^{\ell-1} \frac{\partial h_{j}}{\partial z_{i_{\ell}}} a_{I \backslash i_{\ell}}=b_{j I}^{1} h_{1}+\cdots+b_{j I}^{k} h_{k}, \quad j=1, \ldots, k,
$$

с голоморфными $b_{j I}^{1}, \ldots, b_{j I}^{k} \in \mathscr{O}_{U}$.

Зафиксируем мульти-индекс $J^{p}=\left(j_{1}, \ldots, j_{p}\right), 1 \leqslant j_{1}, \ldots, j_{p} \leqslant m, 1 \leqslant p \leqslant k$, и обозначим соответствующий минор якобиевой матрицы $\operatorname{Jac}\left(h_{1}, \ldots, h_{k}\right)=$ $\left\|\partial h_{i} / \partial z_{j}\right\|$ через

$$
\Delta_{J^{p}}=\Delta_{j_{1} \cdots j_{p}}=\operatorname{det}\left\|\frac{\partial h_{i}}{\partial z_{j_{r}}}\right\|_{1 \leqslant i, r \leqslant p} .
$$

Теперь индукцией по индексу $p$ докажем, что выполняются следующие сравнения:

$$
\Delta_{J^{p}} a_{I^{q}} \equiv \sum_{K \subset I^{q},|K|=p} \operatorname{sgn}\left(\begin{array}{c}
I^{q} \\
K, I^{q} \backslash K
\end{array}\right) \Delta_{K} a_{\left(J^{p}, I^{q} \backslash K\right)}(\bmod (\mathscr{I})), \quad p=1, \ldots, k,
$$


где $\mathscr{I} \subseteq \mathscr{O}_{U}$ - идеал, порожденный регулярной последовательностью $\left(h_{1}, \ldots, h_{k}\right)$. Сначала рассмотрим случай $p=1, J^{1}=j_{1}=j$. Полагая $I=I^{q+1}=\left(j, I^{q}\right)=$ $\left(j, i_{1}, \ldots, i_{q}\right)$ в формуле $(4)$, получаем сравнение

$$
\frac{\partial h_{1}}{\partial z_{j}} a_{I^{q}} \equiv \sum_{\ell=1}^{q}(-1)^{\ell-1} \frac{\partial h_{1}}{\partial z_{i_{\ell}}} a_{I \backslash i_{\ell}}(\bmod (\mathscr{I})),
$$

которое совпадает с соотношением (5) при $p=1$.

Предположим, что сравнение (5) верно при $p-1$, и докажем его справедливость для $p$ таким образом. Разложение детерминанта $\Delta_{J^{p}}$ по $p$-й строке приводит к тождеству

$$
\Delta_{J^{p}} a_{I^{q}}=\sum_{\ell=1}^{p}(-1)^{p-\ell} \frac{\partial h_{p}}{\partial z_{j_{\ell}}} \Delta_{j_{1} \cdots \widehat{j_{\ell} \cdots j_{p}}}^{p-1} a_{I^{q}}
$$

По предположению индукции

$$
\Delta_{j_{1} \cdots \widehat{j_{\ell} \cdots j_{p}}} a_{I^{q}} \equiv \sum_{\substack{K^{\prime} \subset I^{q} \\
\left|K^{\prime}\right|=p-1}} \operatorname{sgn}\left(\begin{array}{c}
I^{q} \\
K^{\prime}, I^{q} \backslash K^{\prime}
\end{array}\right) \Delta_{K^{\prime}} a_{\left(j_{1} \cdots \hat{j_{\ell}} \cdots j_{p}, I^{q} \backslash K^{\prime}\right)}(\bmod (\mathscr{I})) .
$$

Подставляя это соотношение в предыдущее сравнение и меняя порядок суммирования, получаем

$$
\Delta_{J^{p}} a_{I^{q}} \equiv \sum_{\substack{K^{\prime} \subset I^{q} \\
\left|K^{\prime}\right|=p-1}} \operatorname{sgn}\left(\begin{array}{c}
I^{q} \\
K^{\prime}, I^{q} \backslash K^{\prime}
\end{array}\right) \Delta_{K^{\prime}} \sum_{\ell=1}^{p}(-1)^{p-\ell} \frac{\partial h_{p}}{\partial z_{\ell}} a_{\left(j_{1} \cdots \hat{j_{\ell}} \cdots j_{p}, I q K^{\prime}\right)}(\bmod (\mathscr{I})) .
$$

Вторая сумма состоит из $p$ слагаемых, содержащихся в соотношении (4) при $j=p, I=\left(i_{1}, \ldots, i_{p}, I^{q} \backslash K^{\prime}\right)$, а длина этого мульти-индекса равна $q+1$. Следовательно, эту сумму можно переписать в виде суммы остальных $q-p+1$ слагаемых с противоположными знаками и элемента из идеала $\left(h_{1}, \ldots, h_{k}\right) \mathscr{O}_{U}$. Отсюда получается такое сравнение по модулю идеала $\mathscr{I}$ :

$$
\Delta_{J^{p}} a_{I^{q}} \equiv \sum_{\substack{K^{\prime} \subset I^{q} \\
\left|K^{\prime}\right|=p-1}} \operatorname{sgn}\left(\begin{array}{c}
I^{q} \\
K^{\prime}, I^{q} \backslash K^{\prime}
\end{array}\right) \Delta_{K^{\prime}}(-1)^{p-1} \sum_{i \in I \backslash K^{\prime}}(-1)^{\#\left(i ; I \backslash K^{\prime}\right)} \frac{\partial h_{p}}{\partial z_{i}} a_{\left(j_{1} \cdots j_{p}, I^{q} \backslash K^{\prime} \backslash i\right)},
$$

где через \#(i; $\left.\backslash K^{\prime}\right)$ обозначается число вхождений индекса $i$ в множество $I \backslash K^{\prime}$. Упорядочим теперь все пары $\left(K^{\prime}, i\right)$ таким образом, чтобы мульти-индекс $K^{\prime} \cup\{i\}$ совпадал с заданным $K \subset I$. Для любой такой пары соответствующий коэффициент $a_{\left(j_{1} \cdots j_{p}, I \backslash K^{\prime} \backslash i\right)}$ равен $a_{(J, I \backslash K)}$. Тогда вклад упорядоченного выше множества в соотношение (6) будет таким:

$$
\begin{array}{r}
a_{\left(J^{p}, I^{q} \backslash K\right)}(-1)^{p-1} \sum_{i \in K} \operatorname{sgn}\left(\begin{array}{r}
I^{q} \\
K \backslash i, I^{q} \backslash K, i
\end{array}\right)(-1)^{\#(i ; I \backslash K \backslash i)} \frac{\partial h_{p}}{\partial z_{i}} \Delta_{K \backslash i} \\
=\operatorname{sgn}\left(\begin{array}{c}
I^{q} \\
K, I^{q} \backslash K
\end{array}\right) a_{J^{p}, I^{q} \backslash K} \Delta_{K} .
\end{array}
$$

Это завершает доказательство сравнения (5) для $p \geqslant 1$. 
Остается показать, что можно выбрать функцию $g$ таким образом, что $g \not \equiv 0$ на каждой неприводимой компоненте полного пересечения $C$. Для этого рассмотрим идеал $\mathscr{G}$ кольца $\mathscr{O}_{U}$, порожденный всеми минорами $\Delta_{i_{1} \cdots i_{k}}$ максимального порядка якобиевой матрицы $\operatorname{Jac}\left(h_{1}, \ldots, h_{k}\right)$. Поскольку дифференциальная форма $d h_{1} \wedge \cdots \wedge d h_{k}$ не обращается тождественно в нуль на каждой неприводимой компоненте полного пересечения $C$, образ $\widetilde{\mathscr{G}}$ идеала $\mathscr{G}$ в кольце $\mathscr{O}_{C, 0}$ не равен Ann $\mathscr{O}_{C, 0}$. Поэтому можно использовать теорему 2.4(1) работы [5], из которой вытекает, что $\mathscr{O}_{C, 0}$-глубина идеала $\widetilde{\mathscr{G}}$ не меньше единицы. Следовательно, существует элемент $g \in \mathscr{O}_{C}$ с требуемым в теореме 1 свойством.

Замечание 2. Нетрудно проверить, что из формулы (5) следует тождество

$$
\Delta_{i_{1} \cdots i_{k}} \sum_{|I|=q} a_{I} d z_{I}=d h_{1} \wedge \cdots \wedge d h_{k} \wedge\left(\sum_{\left|I^{\prime}\right|=q-k} a_{i_{1} \cdots i_{k} I^{\prime}} d z_{I^{\prime}}\right)+\nu
$$

где $\nu \in \sum_{j=1}^{k} h_{j} \Omega_{U}^{q-k}$. Таким образом, по аналогии со случаем гиперповерхности (см. [13, лемма (2.8)]) максимальные миноры якобиевой матрицы $\mathrm{Jac}\left(h_{1}, \ldots, h_{k}\right)$ могут рассматриваться как универсалъные знаменатели для полных пересечений.

Следствие 1. Пусть $\omega \in \Omega_{X}^{q}(\log D)$ - дифференииальная форма с логарифмическими полюсами вдоль дивизора $D$ и $C=D_{1} \cap \cdots \cap D_{k}-$ полное пересечение. Тогда существует разложение $(3)$ с формой $\eta \in \sum_{i=1}^{k} \Omega_{X}^{\bullet}\left(\log \widehat{D_{i}}\right)$.

Доказательство. Поскольку для логарифмической формы $\omega$ условия (2) выполнены ввиду предложения 1 из $\S 2$, существует разложение (3), в котором $\eta \in \sum_{i=1}^{k} \Omega_{U}^{q}\left(\widehat{D_{i}}\right)$. Рассмотрим случай $k=2$. Тогда $\eta=\eta_{1} / h_{1}+\eta_{2} / h_{2}$, где $\eta_{1}, \eta_{2} \in \Omega_{U}^{q}$. После внешнего умножения на $d h$ обеих частей разложения $(3)$ получаем, что форма $d h \wedge \eta$ голоморфна. Кроме того, поскольку

$$
d h \wedge \eta=d h \wedge\left(\frac{\eta_{1}}{h_{1}}+\frac{\eta_{2}}{h_{2}}\right)=d h_{2} \wedge \eta_{1}+d h_{1} \wedge \eta_{2}+h_{2} \frac{d h_{1}}{h_{1}} \wedge \eta_{1}+h_{1} \frac{d h_{2}}{h_{2}} \wedge \eta_{2},
$$

сумма последних двух слагаемых - также голоморфная форма. Наконец, умножение этих тождеств на общий знаменатель дает включение

$$
h_{2}^{2}\left(d h_{1} \wedge \eta_{1}\right)+h_{1}^{2}\left(d h_{2} \wedge \eta_{2}\right) \in\left(h_{1} h_{2}\right) \Omega_{X}^{\bullet}
$$

т. е. $h_{2}^{2} \alpha+h_{1}^{2} \beta=h_{1} h_{2} \gamma$, где $\alpha, \beta, \gamma \in \Omega_{X}^{\bullet}$, и поэтому $h_{2}^{2} \alpha+\left(h_{1} \beta-h_{2} \gamma\right) h_{1}=0$. Поскольку $\left(h_{1}, h_{2}\right)$ - регулярная последовательность, сравнивая коэффициенты соответствующих форм при фиксированном наборе дифференциалов $d z_{I}$, получаем, что $\alpha=h_{1} \alpha^{\prime}, \alpha^{\prime} \in \Omega_{X}^{\bullet}$. Это означает, что $d h_{1} \wedge \eta_{1} \in\left(h_{1}\right) \Omega_{X}^{\bullet}$, т. е. $\eta_{1} / h_{1} \in \Omega_{X}^{\bullet}\left(\log D_{1}\right)$. Такими же рассуждениями можно проверить, что $\eta_{2} / h_{2} \in \Omega_{X}^{\bullet}\left(\log D_{2}\right)$. Аналогично разбирается и общий случай $k>2$.

Следствие 2. В условиях теоремы 1 разложение (3) имеет место тогда u только тогда, когда существуют аналитические подмножества $A_{j} \subset D_{j}$, $j=1, \ldots, k$, коразмерность которых не менъше 2 , такие, что росток бормы $\omega$ в любой точке $x \in \bigcup_{j=1}^{k}\left(D_{j} \backslash A_{j}\right)$ принадлежит пространству

$$
\frac{d h_{1}}{h_{1}} \wedge \cdots \wedge \frac{d h_{k}}{h_{k}} \wedge \Omega_{U, x}^{q-k}+\sum_{i=1}^{k} \Omega_{U, x}^{q}\left(\widehat{D_{i}}\right) .
$$


Доказательство. В одну сторону доказательство очевидно: следует положить $A_{j}=D_{j} \cap\{g=0\}, j=1, \ldots, k$, и использовать разложение из теоремы 1.

Обратное утверждение получается такими рассуждениями. Если для мероморфной формы $\omega$ существует представление (7), то $h \omega$ голоморфна вне подмножеств $A_{i} \subset D_{i}, i=1, \ldots, k$, коразмерности, не меньшей 2. Следовательно, по теореме продолжения Римана дифференциальная форма $h \omega$ голоморфна всюду, так что $h_{j} \omega \in \Omega_{U}^{q}\left(\widehat{D_{j}}\right), j=1, \ldots, k$. Далее, $d h_{j} \wedge \omega$ выражается в виде суммы мероморфных форм $\omega_{i}$, каждая из которых особа не более чем на $k-1$ компонентах дивизора $\widehat{D_{i}}$ и на подмножестве $A_{i} \subset D_{i}$ коразмерности, не меньшей 2. Снова, применяя теорему продолжения Римана к $\left(h_{1} \cdots \widehat{h_{i}} \cdots h_{k}\right) \omega_{i}$, получаем, что форма $\omega_{i}$ имеет особенности только на $\widehat{D_{i}}$. В результате получаем, что $d h_{j} \wedge \omega \in \sum_{i=1}^{k} \Omega_{U}^{q}\left(\widehat{D_{i}}\right)$ для всех $j=1, \ldots, k$.

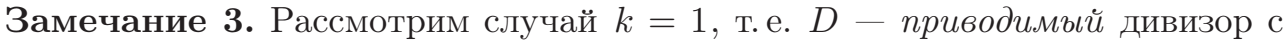
несократимым разложением длины единица. Тогда $C=D$ и представление (3) принимает вид

$$
g \omega=\frac{d h}{h} \wedge \xi+\eta, \quad \xi, \eta \in \Omega_{U}^{\bullet} .
$$

Таким образом, разложение (3) совпадает с представлением (iii) из основной леммы Саито (см. [13, (1.1)]).

\section{§4. Кратный вычет мероморфных форм}

Обсудим теперь понятие кратного вычета мероморфных форм, удовлетворяющих условиям $\S 3$. Сначала заметим, что функция $g$ из разложения (3)это неделитель нуля в локальном кольце $\mathscr{O}_{X, x} /\left(h_{1}, \ldots, h_{k}\right) \mathscr{O}_{X, x} \cong \mathscr{O}_{C, x}$. Поэтому можно рассмотреть ограничение формы $\xi / g$ на росток полного пересечения $C=D_{1} \cap \cdots \cap D_{k}$.

Определение 1. Ограничение дифференциальной формы $\xi / g$ на полное пересечение $C$ называется формой кратного вычета или кратным вычетом дифференциальной формы $\omega$ и обозначается через

$$
\operatorname{Res}_{C}(\omega)=\left.\frac{\xi}{g}\right|_{C} .
$$

Таким образом, дифференциальная форма кратного вычета принадлежит пространству $\mathscr{M}_{C} \otimes_{\mathscr{O}_{C}} \Omega_{C}^{q-k} \cong \mathscr{M}_{\widetilde{C}} \otimes_{\mathscr{O}_{\widetilde{C}}} \Omega_{\widetilde{C}}^{q-k}, q \geqslant k$, где $\widetilde{C}-$ нормализация $C$, а $\mathscr{M}_{C}$ и $\mathscr{M}_{\widetilde{C}}-$ квазикогерентные пучки мероморфных функций на $C$ и $\widetilde{C}$ соответственно.

Предложение 2. Кратный вычет определен корректно, т.е. определяемая им дифференциальная форма не зависит от выбора представления (3).

Доказательство. Предположим, что $q \geqslant k$ и $q$-форма $\omega$ обладает двумя локальными представлениями

$$
g_{\ell} \omega=\frac{d h_{1}}{h_{1}} \wedge \cdots \wedge \frac{d h_{k}}{h_{k}} \wedge \xi_{\ell}+\eta_{\ell}, \quad \ell=1,2 .
$$

Тогда $d h_{1} \wedge \cdots \wedge d h_{k} \wedge\left(g_{1} \xi_{2}-g_{2} \xi_{1}\right)=h_{1} \cdots h_{k}\left(g_{1} \eta_{2}-g_{2} \eta_{1}\right) \in\left(h_{1}, \ldots, h_{k}\right) \Omega_{X}^{q}$, так что

$$
d h_{1} \wedge \cdots \wedge d h_{k} \wedge\left(g_{1} \xi_{2}-g_{2} \xi_{1}\right) \equiv 0\left(\bmod \left(h_{1}, \ldots, h_{k}\right)\right) .
$$


Из утверждения і) теоремы из статьи [12] (вариант обобщенной леммы де Рама) при $R=\mathscr{O}_{C, x}, M=\Omega_{X, x}^{1} \otimes \mathscr{O}_{C, x}, e_{i}=z_{i}, i=1, \ldots, m, \omega_{j}=d h_{j}, j=1, \ldots, k$, $p=q-k \geqslant 0$, следует, что

$$
\mathscr{G}^{e}\left(g_{1} \xi_{2}-g_{2} \xi_{1}\right) \subset d h_{1} \wedge \Omega_{X, x}^{q-k-1}+\cdots+d h_{k} \wedge \Omega_{X, x}^{q-k-1}+\left(h_{1}, \ldots, h_{k}\right) \Omega_{X, x}^{q-k}, \quad e \in \mathbf{Z}_{+},
$$

где идеал $\mathscr{G} \subset \mathscr{O}_{X, x}$ порождается всеми минорами $\Delta_{i_{1} \cdots i_{k}}$ максимального порядка якобиевой матрицы $\mathrm{Jac}\left(h_{1}, \ldots, h_{k}\right)$. Как и в конце доказательства теоремы 1 , заметим, что, поскольку росток $C$ приведен, образ $\widetilde{\mathscr{G}}$ идеала $\mathscr{G}$ в кольце $\mathscr{O}_{C, x}$ не равен Ann $\mathscr{O}_{C, x}$, а потому $\mathscr{O}_{C, x}$-глубина идеала $\widetilde{\mathscr{G}}$ не меньше единицы. Следовательно, найдется элемент $\Delta \in \widetilde{\mathscr{G}}$, неделитель нуля в $\mathscr{O}_{C, x}$, такой, что

$$
\Delta^{e}\left(g_{1} \xi_{2}-g_{2} \xi_{1}\right) \in d h_{1} \wedge \Omega_{X, x}^{q-k-1}+\cdots+d h_{k} \wedge \Omega_{X, x}^{q-k-1}+\left(h_{1}, \ldots, h_{k}\right) \Omega_{X, x}^{q-k} .
$$

Поэтому класс элемента $\Delta^{e}\left(g_{1} \xi_{2}-g_{2} \xi_{1}\right)$ в $\Omega_{C, x}^{q-k}$ равен нулю. Это и означает, что два элемента $\frac{1}{g_{1}} \xi_{1}$ и $\frac{1}{g_{2}} \xi_{2}$ определяют один и тот же класс в $\mathscr{M}_{C, x} \otimes_{\mathscr{O}_{C, x}} \Omega_{C, x}^{q-k}$.

Лемма 1. Ядро отображения кратного вычета $\operatorname{Res}_{C}$ совпадает с пространством $\sum_{i=1}^{k} \Omega_{X}^{\bullet}\left(\widehat{D_{i}}\right)$.

Доказательство. Очевидно, что ядро содержит указанную сумму. Докажем обратное включение. Итак, пусть $q \geqslant k$ и $\operatorname{Res}_{C}(\omega)=0$ для некоторой $q$-формы $\omega$. Тогда существует функция $g$ в разложении (3) теоремы 1 , такая, что ограничение мероморфной регулярной формы $\xi / g$ на росток пространства $C$ обращается в нуль. Следовательно, $\xi=g\left(\sum h_{i} \xi_{i}+\sum d h_{i} \wedge \xi_{i}^{\prime}\right)$, где $\xi_{i}, \xi_{i}^{\prime} \in \Omega_{X}^{\bullet}$, и

$$
h \omega=d h_{1} \wedge \cdots \wedge d h_{k} \wedge\left(\sum h_{i} \xi_{i}\right)+\frac{1}{g}\left(\sum h_{i} \eta_{i}\right), \quad \eta_{i} \in \Omega_{X}^{\bullet} .
$$

Так как $h \omega$ и первое слагаемое в правой части тождества голоморфны, то $g$ делит $\sum h_{i} \eta_{i}$ в $\Omega_{X}^{\bullet}$, т. е. $g \eta_{0}=\sum h_{i} \eta_{i}, \eta_{0} \in \Omega_{X}^{\bullet}$. С другой стороны, $\left(h_{1}, \ldots, h_{k}\right)$ - регулярная последовательность, а $g$ - неделитель нуля в локальной алгебре $\mathscr{O}_{C}=\mathscr{O}_{X} /\left(h_{1}, \ldots, h_{k}\right) \mathscr{O}_{X}$. Поэтому, рассматривая коэффициенты голоморфной формы $\sum h_{i} \eta_{i}$ при каждом фиксированном наборе дифференциалов $d z_{I}$, получаем, что $\eta_{0} \in\left(h_{1}, \ldots, h_{k}\right) \Omega_{X}^{\bullet}$. Отсюда следует, что $\omega \in \sum_{i=1}^{k} \Omega_{X}^{\bullet}\left(\widehat{D_{i}}\right)$.

Замечание 4. Если $m=k$, т.е. $\operatorname{dim} C=0$ и пространство $C \neq C_{\text {red }}$ не приведено, то тождество из замечания 2 справедливо при $\nu=0$. В частности, из этого следует, что существует представление (3) с функцией $g$, заданной некоторым элементом одномерного цоколя локальной алгебры $\mathscr{O}_{C}$. Хорошо известно, что для 0 -мерного полного пересечения цоколь порождается над основным полем детерминантом якобиевой матрицы Jac $(h)$ (см. [15]), поскольку $d h_{1} \wedge \cdots \wedge d h_{k}=\Delta_{i_{1} \cdots i_{k}} d z_{1} \wedge \cdots \wedge d z_{m}$. Нетрудно понять, что в этом случае кратный вычет стариих дифференциальных $m$-форм совпадает с так называемым многомерным вычетом, который в контексте теории локальной двойственности Гротендика выражается в терминах проекции элементов некоторого конечномерного пространства на этот цоколь (см. [7]). Поэтому, как в формулировке теоремы 1 , так и далее, можно предполагать, что $C=C_{\mathrm{red}}$, поскольку неприведенный нульмерный случай хорошо исследован и подробно описан в литературе. 


\section{§5. Регулярные мероморфные дифференциальные формы}

Пусть $X-$ комплексное многообразие, $\operatorname{dim} X=m \geqslant 1$, и аналитическое подмножество $Y \subset X$ в окрестности точки $x \in U \subset X$ задается набором функций $f_{1}, \ldots, f_{k} \in \mathscr{O}_{U}$. Обозначим через $\Omega_{Y}^{q}, q \geqslant 0$, пучки ростков регулярных голоморфных дифференциальных $q$-форм на $Y$, которые задаются как ограничение на $Y$ фактормодуля

$$
\Omega_{U}^{q} /\left.\left(\left(f_{1}, \ldots, f_{k}\right) \Omega_{U}^{q}+d f_{1} \wedge \Omega_{U}^{q-1}+\cdots+d f_{k} \wedge \Omega_{U}^{q-1}\right)\right|_{Y} .
$$

Тогда обычный дифференциал $d$ наделяет это семейство пучков структурой комплекса, который называется комплексом де Рама на $Y$ и обозначается через $\left(\Omega_{Y}^{\bullet}, d\right)$.

В этом параграфе будем предполагать, что $Y$ - пространство Коэна-Маколея и $\operatorname{dim} Y=n$. Напомним, что $\mathscr{O}_{Y}$-модуль

$$
\omega_{Y}^{n}=\operatorname{Ext}_{\mathscr{O}_{X}}^{m-n}\left(\mathscr{O}_{Y}, \Omega_{X}^{m}\right)
$$

называется дуализирующим модулем Гротендика пространства $Y$.

Определение 2. Когерентный пучок $\mathscr{O}_{Y}$-модулей $\omega_{Y}^{q}, q \geqslant 0$, локально задается как множество ростков мероморфных форм $\omega$ степени $q$ на $Y$, таких, что $\omega \wedge \eta \in \omega_{Y}^{n}$ для любой $\eta \in \Omega_{Y}^{n-q}$. Другими словами (см. [4]),

$$
\omega_{Y}^{q} \cong \operatorname{Hom}_{\mathscr{O}_{Y}}\left(\Omega_{Y}^{n-q}, \omega_{Y}^{n}\right) \cong \operatorname{Ext}_{\mathscr{O}_{X}}^{m-n}\left(\Omega_{Y}^{n-q}, \Omega_{X}^{m}\right) .
$$

Элементы из $\omega_{Y}^{q}$ называются регулярными мероморфными дифференциальными формами степени $q$ на $Y$. Известны и другие способы описания таких форм: в терминах нормализации Нётер и следа (см. [10], [4]), в терминах вычетных потоков (см. [2]) и т. д.

Вот некоторые полезные свойства регулярных мероморфных дифференциальных форм:

1) $\omega_{Y}^{q}=0$, если $q<0$ или $q>\operatorname{dim} Y$;

2) $\omega_{Y}^{q}$ не имеет кручения, т. е. Tors $\omega_{Y}^{q}=0, q \geqslant 0$;

$3)$ дифференциал де Рама $d$, действующий на $\Omega_{Y}^{q}$, продолжается на семейство модулей $\omega_{Y}^{q}, 0 \leqslant q \leqslant n$, и наделяет это семейство структурой комплекса $\left(\omega_{Y}^{\bullet}, d\right)$;

4) существуют вложения $\omega_{Y}^{q} \subseteq j_{*} j^{*} \Omega_{Y}^{q}, q \geqslant 0$, где $j: Y \backslash Z \longrightarrow Y$ - каноническое вложение и $Z=\operatorname{Sing} Y$, причем, если $Y-$ нормальное пространство, то $\omega_{Y}^{q} \cong j_{*} j^{*} \Omega_{Y}^{q}$

5) если $\pi: \widetilde{Y} \rightarrow Y-$ (конечный) морфизм нормализации ростка пространства $Y$, то отображение прямого образа $\pi_{*}: \omega_{\widetilde{Y}}^{\bullet} \rightarrow \omega_{Y}^{\bullet}$ инъективно; если к тому же росток нормализации гладкий и коразмерность множества точек, в окрестности которых $\pi$ - локальный изоморфизм, не меньше двух, то отображение $\pi_{*}$ - эпиморфизм (см. [4]), и это означает, что $\omega_{\widetilde{Y}}^{\bullet}$ и $\omega_{Y}^{\bullet}$ изоморфны и, в частности, $\omega_{Y}^{\bullet} \cong \Omega_{\widetilde{Y}}^{\bullet}$

$6)$ если $Y$ - простая рациональная особенность типа $A_{k}, D_{k}, E_{6}, E_{7}$ или $E_{8}$, то комплекс $\left(\omega_{Y}^{\bullet}, d\right)$ ацикличен в положительных размерностях (см. [9, замечание $(4.8)(2)])$, т. е. $\omega_{Y}^{\bullet}$ - это резольвента постоянного пучка $\mathbb{C}_{Y}$.

Предположим теперь, что $Y=C$ - полное пересечение, которое в окрестности $U$ точки $x \in C$ задается регулярной последовательностью функций $f_{1}, \ldots, f_{k}$ 
$\in \mathscr{O}_{U}$. Тогда $n=m-k$ и

$$
\omega_{C, x}^{n}=\operatorname{Ext}_{\mathscr{O}_{X, x}}^{k}\left(\mathscr{O}_{C, x}, \Omega_{X, x}^{m}\right) \cong \mathscr{O}_{C, x}\left(d z_{1} \wedge \cdots \wedge d z_{m} / d f_{1} \wedge \cdots \wedge d f_{k}\right) .
$$

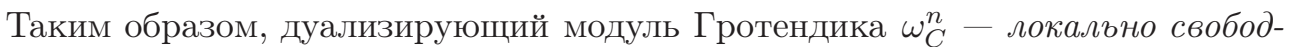
ный $\mathscr{O}_{C}$-модуль ранга единица. Кроме того, существуют изоморфизмы $\mathscr{O}_{X}$-модулей

$$
\omega_{C}^{q} \cong \operatorname{Hom}_{\mathscr{O}_{C}}\left(\Omega_{C}^{n-q}, \mathscr{O}_{C}\right) \cong \operatorname{Ext}_{\mathscr{O}_{X}}^{k}\left(\Omega_{C}^{n-q}, \mathscr{O}_{X}\right), \quad 0 \leqslant q \leqslant n .
$$

\section{§6. Кратный вычет и логарифмические формы}

Как уже отмечалось (ср. следствие 1), для логарифмических форм с полюсами вдоль дивизора, удовлетворяющего предположениям из §3, существует разложение (3) и, следовательно, корректно определено ограничение кратного вычета $\operatorname{Res}_{C}$ на соответствующее подпространство мероморфных дифференциальных форм.

Лемма 2. В условиях $§ 3$ пусть $\omega \in \Omega_{X}^{q}(\log D)$ - дифберенииальная форма с логарифмическими полюсами вдоль гиперповерхности $D$ u $C=D_{1} \cap \cdots \cap D_{k}$ - полное пересечение. Тогда отображсение кратного вычета совместимо с действием дифберенииала де Рама.

Доказательство. Применим оператор дифференцирования $d \mathrm{k}$ представлению (3) формы $\omega$ :

$$
\omega=\frac{d h_{1}}{h_{1}} \wedge \cdots \wedge \frac{d h_{k}}{h_{k}} \wedge \frac{\xi}{g}+\frac{\eta}{g} .
$$

Тогда форма $\operatorname{Res}_{C}(d \omega)$ и ограничение дифференциала $d\left(g^{-1} \xi\right)$ на $C$ равны (с точностью до знака).

Утверждение следующей теоремы в случае $k=1$ получено в работе [1].

Теорема 2. В условиях теоремь 1 пусть $C=D_{1} \cap \cdots \cap D_{k}-$ приведенное полное пересечение. Тогда при $p \geqslant k$ существует точная последовательность

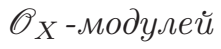

$$
0 \longrightarrow \sum_{i=1}^{k} \Omega_{X}^{p}\left(\log \widehat{D_{i}}\right) \longrightarrow \Omega_{X}^{p}(\log D) \stackrel{\operatorname{Res}_{C}}{\longrightarrow} \omega_{C}^{p-k} \longrightarrow 0
$$

Доказательство. Сначала вычислим ядро ограничения морфизма кратного вычета $\operatorname{Res}_{C}$ на $\Omega_{X}^{\bullet}(\log D)$. Ввиду утверждения 3 из $\S 2$ и леммы 1 из $\S 4$ для этого достаточно проверить, что

$$
\Omega_{X}^{\bullet}(\log D) \cap \Omega_{X}^{\bullet}\left(\widehat{D_{j}}\right)=\Omega_{X}^{\bullet}\left(\log \widehat{D_{j}}\right)
$$

для всех $j=1, \ldots, k$. Поскольку $\Omega_{X}^{\bullet}\left(\log \widehat{D_{j}}\right) \subseteq \Omega_{X}^{\bullet}(\log D)$, правая часть содержится в левой. Для доказательства обратного включения разберем подробно случай $k=2$. Итак, пусть $\omega \in \Omega_{X}^{\bullet}\left(\widehat{D_{1}}\right)=\Omega_{X}^{\bullet}\left(D_{2}\right) \cong \frac{1}{h_{2}} \Omega_{X}^{\bullet}$, т. е. $\omega=\xi / h_{2}$. Если $\omega \in \Omega_{X}^{\bullet}(\log D)$, то $h \omega=h_{1} \xi \in \Omega_{X}^{\bullet}$ и

$$
d h \wedge \omega=d h_{1} \wedge \xi+d h_{2} \wedge\left(h_{1} \omega\right) \in \Omega_{X}^{\bullet} .
$$

Отсюда следует, что $d h_{2} \wedge\left(h_{1} \omega\right) \in \Omega_{X}^{\bullet}$, т. е. $d h_{2} \wedge\left(h_{1} \xi\right) \in\left(h_{2}\right) \Omega_{X}^{\bullet}$. Поэтому $h_{1} d h_{2} \wedge \xi=h_{2} \eta$, где $\eta \in \Omega_{X}^{\bullet}$. Так как $h_{1}, h_{2}$ - регулярная последовательность, то, сравнивая коэффициенты форм $d h_{2} \wedge \xi$ и $\eta$, получаем, что $h_{1}$ делит $\eta$ и, следовательно, $d h_{2} \wedge \xi \in\left(h_{2}\right) \Omega_{X}^{\bullet}, d h_{2} \wedge \omega \in \Omega_{X}^{\bullet}$, т. е. $\omega \in \Omega_{X}^{\bullet}\left(D_{2}\right)=\Omega_{X}^{\bullet}\left(\widehat{D_{1}}\right)$. 
Это и требовалось установить. Общий случай $k \geqslant 2$ разбирается аналогично. Теперь для описания образа морфизма $\operatorname{Res}_{C}$ нужно почти дословно повторить рассуждения из $[1, \S 4]$ в случае $k=1$, следуя доказательству теоремы из работы [2].

Замечание 5. В обозначениях замечания 3 пусть $k=1$ и $C=D$. Тогда $\operatorname{Res}_{C}=\operatorname{Res}_{D}-$ это отображение вычета, которое описано в [13] и которое обозначается там через res. Далее, в этом случае существуют точные последовательности (см. [1])

$$
0 \longrightarrow \Omega_{X}^{q} \longrightarrow \Omega_{X}^{q}(\log D) \stackrel{\operatorname{Res}_{D}}{\longrightarrow} \omega_{D}^{q-1} \longrightarrow 0, \quad q \geqslant 1,
$$

пополняющие справа диаграмму (2.5) из работы [13]. Таким образом, в теореме 2 получены аналогичные последовательности для отображения кратного вычета.

Следствие 3. При тех же предположениях существует естественный изоморфизм

$$
\mathscr{H}_{D R}^{p}\left(\Omega_{X}^{\bullet}(\log D)\right) \cong \mathscr{H}_{D R}^{p-1}\left(\omega_{D}^{\bullet}\right),
$$

где $\mathscr{H}_{D R}^{*}$ - функтор когомологий комплексов с дифференииалом де Рама d. $B$ частности, $\Omega_{X}^{\bullet}(\log D)$ ацикличен в размерностях $p>1$, если $D-$ простая рачиональная особенность типа $A_{k}, D_{k}, E_{6}, E_{7}$ или $E_{8}$ размерности $n \geqslant 2$.

Доказательство. Поскольку отображение вычета совместимо с дифференциалом $d$, последовательности (9) индуцируют точную последовательность соответствующих комплексов. Отсюда получаются требуемые изоморфизмы. Далее, как уже отмечалось в $\S 5$ (см. свойство 6$)$ ), для рациональных особенностей указанных типов комплекс $\left(\omega_{D}^{\bullet}, d\right)$ ацикличен в положительных размерностях; это дает вторую часть утверждения. Кроме того, так как размерность пространства $\mathscr{H}_{D R}^{0}\left(\omega_{D}^{\bullet}\right)$ равна числу неприводимых компонент дивизора $D$ (см. $[9$, $(4.1)])$, то $\mathscr{H}_{D R}^{1}\left(\Omega_{X}^{\bullet}(\log D)\right) \cong \mathbb{C}$ при $n \geqslant 2$. Для полноты отметим, что утверждения следствия можно получить и непосредственно с помощью вычислений (cм. [8]).

В заключение этого параграфа рассмотрим важный пример, когда образ кратного вычета порождается голоморфными формами. Обозначим через $\Omega_{X}^{p}\langle D\rangle$ $\subseteq \Omega_{X}^{p}(\log D)$ пространство логарифмических $p$-форм главного типа, так что

$$
\Omega_{X}^{p}\langle D\rangle=\bigwedge^{p}\left\langle\frac{d h_{1}}{h_{1}}, \ldots, \frac{d h_{k}}{h_{k}}, d z_{1}, \ldots, d z_{m}\right\rangle .
$$

Легко видеть, что семейство $\Omega_{X}^{p}\langle D\rangle, p \geqslant 0$, образует подкомплекс $\Omega_{X}^{\bullet}\langle D\rangle$ логарифмического комплекса де Рама $\Omega_{X}^{\bullet}(\log D)$, замкнутый относительно внешнего дифференцирования и внешнего умножения на $d h_{i} / h_{i}, 1 \leqslant i \leqslant k$. Далее, в разложении из теоремы 1 для любой формы $\omega \in \Omega_{X}^{p}\langle D\rangle, p \geqslant k$, можно в качестве функции $g$ выбрать подходящий обратимый элемент. Поэтому кратный вычет такой формы голоморфен, т. е. $\operatorname{Res}_{C}(\omega) \in \Omega_{C}^{p-k}$. Аналогично, если дивизор $D$ таков, что для некоторого $p \geqslant k$ существует изоморфизм $\Omega_{X}^{p}\langle D\rangle \cong \Omega_{X}^{p}(\log D)$, то в этом случае образ отображения кратного вычета порождается голоморфными формами степени $p-k$. Такие дивизоры (с некоторыми дополнительными условиями на пересечения компонент) используются при изучении когомологий «скрученного» комплекса де Рама (см. [11]). 


\section{§7. Весовая фильтрация}

Перейдем теперь к описанию весовой фильтрации на логарифмическом комплексе де Рама для дивизоров, неприводимые компоненты которых локально задаются регулярной последовательностью голоморфных функций.

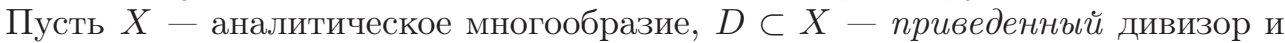
$D=D_{1} \cup \cdots \cup D_{k}$ - его разложение на неприводимые компоненты. Простоты ради будем также считать, что $D_{i}, i=1, \ldots, k$, не имеют самопересечений. Для произвольного упорядоченного набора $I=\left(i_{1} \cdots i_{n}\right), 1 \leqslant i_{1}<\cdots<i_{n} \leqslant k$, длины $n=\#(I)$ рассмотрим следующие ростки:

$$
D_{I}=D_{\left(i_{1} \cdots i_{n}\right)}=D_{i_{1}} \cup \cdots \cup D_{i_{n}}, \quad C^{I}=C^{\left(i_{1} \cdots i_{n}\right)}=D_{i_{1}} \cap \cdots \cap D_{i_{n}} .
$$

Обозначим через $C^{(n)}$ росток аналитического подпространства многообразия $X$, заданный объединениями ростков $C^{\left(i_{1} \cdots i_{n}\right)}$ по всем допустимым наборам, так что $C^{(1)}=D, C^{(k)}=C^{\left(i_{1} \cdots i_{k}\right)}=C$ и т. д. Кроме того, удобно полагать $D_{0}=C^{0}=\varnothing$.

Определение 3. Весовая фильтрачия, или фильтрачия весов, $W$ на логарифмическом комплексе де Рама $\Omega_{X}^{\bullet}(\log D)$ локально задается следующим образом:

$$
W_{n}\left(\Omega_{X, x}^{p}(\log D)\right)= \begin{cases}0, & n<0 \\ \Omega_{X, x}^{p}, & n=0, \\ \sum_{\#(I)=p} \Omega_{X, x}^{p}\left(\log D_{I}\right), & n \geqslant p, 0<p<k_{x}, \\ \sum_{\#(I)=n}^{p} \Omega_{X, x}^{p}\left(\log D_{I}\right), & \text { в оставшихся случаях, }\end{cases}
$$

где через $k_{x}$ обозначается число неприводимых компонент дивизора $D$, проходящих через точку $x \in X$. Например, первые нетривиальные элементы весовой фильтрации при $k=3$ можно представить в виде такой таблицы:

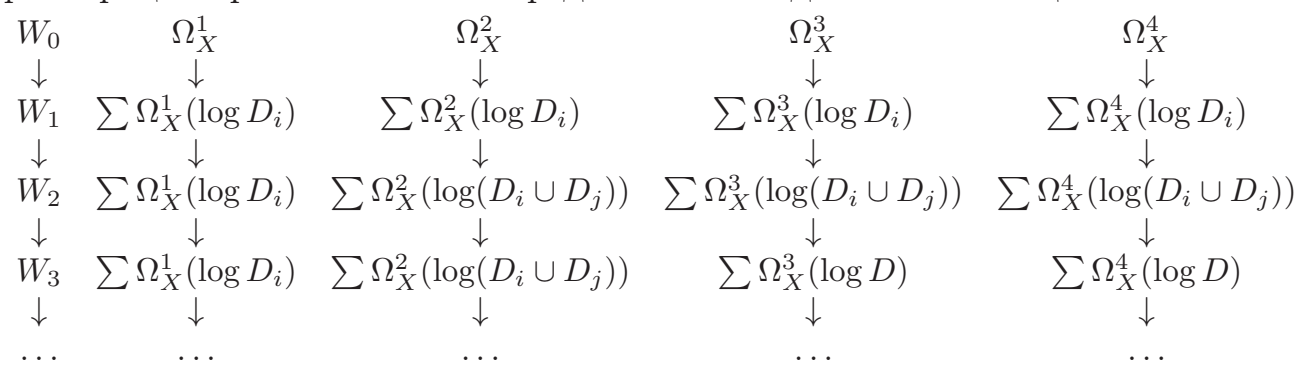

Таким образом, $W_{n}\left(\Omega_{X, x}^{p}(\log D)\right)=\Omega_{X, x}^{p}(\log D)$, если $n \geqslant p \geqslant k_{x}$. Далее, $W$ - возрастающая фильтрация и ввиду $d$ - и $\wedge$-замкнутости внешней алгебры $\Omega_{X}^{\bullet}(\log D)$ для всех целых $p, q, n, \ell$ существуют естественные вложения

$$
\begin{gathered}
d\left(W_{n}\left(\Omega_{X}^{\bullet}(\log D)\right)\right) \subset W_{n}\left(\Omega_{X}^{\bullet}(\log D)\right), \\
W_{n}\left(\Omega_{X}^{p}(\log D)\right) \wedge W_{\ell}\left(\Omega_{X}^{q}(\log D)\right) \subset W_{n+\ell}\left(\Omega_{X}^{p+q}(\log D)\right) .
\end{gathered}
$$

Отметим, что при $n \leqslant p$ пространство $W_{n}\left(\Omega_{X, x}^{p}(\log D)\right)$ содержит все дифференциальные формы вида

$$
\frac{d h_{i_{1}}}{h_{i_{1}}} \wedge \cdots \wedge \frac{d h_{i_{\ell}}}{h_{i_{\ell}}} \wedge \Omega_{X, x}^{p-\ell}, \quad 1 \leqslant i_{1}<\cdots<i_{\ell} \leqslant k, 1 \leqslant \ell \leqslant n,
$$


где $h_{i_{1}}, \ldots, h_{i_{\ell}}$ - локальные уравнения компонент дивизора $D$, содержащих точку $x \in X$. Кроме того,

$$
\Omega_{X, x}^{n}\langle D\rangle \wedge \Omega_{X, x}^{p-n} \subseteq W_{n}\left(\Omega_{X, x}^{p}(\log D)\right) \subseteq \Omega_{X, x}^{n}(\log D) \wedge \Omega_{X, x}^{p-n}, \quad n \in \mathbb{Z}, p \geqslant n .
$$

В случае дивизора с нормальными пересечениями $\Omega_{X}^{\bullet}\langle D\rangle=\Omega_{X}^{\bullet}(\log D)$. Поэтому оба включения в последней формуле превращаются в равенства, а весовая фильтрация на комплексе $\Omega_{X}^{\bullet}\langle D\rangle$ задается так:

$$
W_{n}\left(\Omega_{X}^{p}\langle D\rangle\right)=\Omega_{X}^{n}\langle D\rangle \wedge \Omega_{X}^{p-n}, \quad n \in \mathbf{Z} .
$$

Следующее утверждение можно рассматривать как обобщение изоморфизма (3.1.5.2) из работы [6] для дивизора с нормальными пересечениями на случай дивизора, компоненты которого задаются регулярной последовательностью функций.

Пусть $\pi: \widetilde{C}^{(n)} \rightarrow C^{(n)}$ - морфизм нормализации, так что $\widetilde{C}^{(n)}-$ это несвязная сумма нормализаций $\widetilde{C}^{\left(i_{1} \cdots i_{n}\right)}$ для всех наборов длины $n \geqslant 1$. Обозначим через $\iota$ проекцию $\widetilde{C}^{(n)}$ в $X$, так что $\iota=i \circ \pi$, где $i: C^{(n)} \rightarrow X$ - естественное вложение.

Теорема 3. Предположим, что дивизор D удовлетворяет условиям теоремъ 1 и морбизм нормализации индуиирует изоморфизм комплексов

$$
\pi_{*}: \omega_{\widetilde{C}^{(n)}}^{\bullet} \cong \omega_{C^{(n)}}^{\bullet} .
$$

Тогда отображсние кратного вычета

$$
\operatorname{Res}_{n}^{\bullet}: W_{n}\left(\Omega_{X}^{\bullet}(\log D)\right) \longrightarrow \iota_{*} \omega_{\widetilde{C}^{(n)}}^{\bullet}[-n],
$$

индуиирует изоморфизм комплексов $\mathscr{O}_{X}$-модулей

$$
\operatorname{Gr}_{n}^{W}\left(\Omega_{X}^{\bullet}(\log D)\right) \cong \iota_{*} \omega_{\widetilde{C}^{(n)}}^{\bullet}[-n] .
$$

Доказательство. Сначала отметим, что морфизм нормализации индуцирует изоморфизм $\pi_{*}$, если выполнены условия 5) из §5. Далее, наше утверждение достаточно доказать локально, для ростка $(X, x)$ и для всех $n \leqslant p$. Для любого упорядоченного набора $I=\left(i_{1} \cdots i_{n}\right), 1 \leqslant i_{1}<\cdots<i_{n} \leqslant k$, согласно теореме 2 , при $D=D_{I}$ существует точная последовательность комплексов $\mathscr{O}_{X, x}$-модулей

$$
0 \longrightarrow \sum_{\ell=1}^{n} \Omega_{X, x}^{\bullet}\left(\log \left(\widehat{D_{I}}\right)_{i_{\ell}}\right) \longrightarrow \Omega_{X, x}^{\bullet}\left(\log D_{I}\right) \stackrel{\operatorname{Res}_{C^{I}}}{\longrightarrow} \omega_{C^{I}, x}^{\bullet}[-n] \longrightarrow 0
$$

Из основных свойств регулярных мероморфных дифференциальных форм следует, что $\omega_{\widetilde{C}^{(n)}}^{\bullet}$ изоморфен прямой сумме комплексов $\omega_{\widetilde{C}^{I}}^{\bullet}$ по всем допустимым наборам $I=\left(i_{1} \cdots i_{n}\right)$. Далее, любая форма $\omega \in W_{n}\left(\Omega_{X}^{\bullet}(\log D)\right)$ разлагается в сумму элементов $\omega_{I} \in \Omega_{X}^{\bullet}\left(\log D_{I}\right)$. Определим $\operatorname{Res}_{C}^{(n)}(\omega)$ как сумму вычетов $\operatorname{Res}_{C^{I}}\left(\omega_{I}\right)$. В итоге получаем точную последовательность $\mathscr{O}_{X}$-модулей

$$
0 \longrightarrow W_{n-1}\left(\Omega_{X}^{\bullet}(\log D)\right) \longrightarrow W_{n}\left(\Omega_{X}^{\bullet}(\log D)\right) \stackrel{\operatorname{Res}_{C}^{(n)}}{\longrightarrow} \iota_{*} \omega_{\widetilde{C}^{(n)}}^{\bullet}[-n] \longrightarrow 0 .
$$

Отсюда теперь следует и существование изоморфизмов теоремы 3.

Следствие 4. В условиях теоремы 3 существуют естественные изоморфизмы пространств когомологий

$$
\mathscr{H}^{i}\left(\operatorname{Gr}_{n}^{W}\left(\Omega_{X}^{\bullet}(\log D)\right)\right) \cong \mathscr{H}^{i}\left(\omega_{\widetilde{C}^{(n)}}^{\bullet}\right)[-n]
$$


npu $i \geqslant 1$ u $1 \leqslant n \leqslant k$.

Доказательство. Так как нормализация $\pi$ - конечный, а потому и афбинный морфизм, то

$$
\mathscr{H}^{i}\left(\pi_{*} \omega_{\widetilde{C}^{(n)}}^{\bullet}\right) \cong \mathscr{H}^{i}\left(\omega_{\widetilde{C}^{(n)}}^{\bullet}\right), \quad i \geqslant 1
$$

и нужное утверждение следует из предыдущей теоремы.

Замечание 6. Анализируя общий случай, когда комплексы $\omega_{\widetilde{C}^{(n)}}^{\bullet}$ и $\omega_{C^{(n)}}^{\bullet}$ не изоморфны, в формулировке теоремы 3 соответствующие отображения следует заменить на эпиморфизмы.

Замечание 7. Пусть на многообразии $X$ действует (конечная) группа $G$. Тогда нетрудно показать, что отображение вычета $\operatorname{Res}_{C}$ совместимо с действием этой группы в обычном смысле. В этом случае комплекс пучков регулярных мероморфных форм $\omega_{X / G}^{\bullet}$ на фактормногообразии $X / G$ является резольвентой постоянного пучка [9]. Пользуясь простейшими свойствами пучков $\Omega_{X}^{p}(\log D)$, $\omega_{C}^{q}$ и соответствующих подпучков, инвариантных относительно действия группы $G$, получаем изоморфизм леммы (1.19) из работы [14] для дивизоров с нормальными пересечениями на $V$-многообразии.

Рассмотрим теперь одно приложение. Каноническая убъвающая фильтрация Ходжа $F$ на логарифмическом комплексе де Рама $\Omega_{X}^{\bullet}(\log D)$ определяется следующим образом:

$$
F^{n}\left(\Omega_{X}^{p}(\log D)\right)= \begin{cases}\Omega_{X}^{p}(\log D), & n \leqslant p \\ 0, & n>p .\end{cases}
$$

Предположим теперь, что естественные вложения

$$
\sum_{\#(I)=p} \Omega_{X, x}^{p}\left(\log D_{I}\right) \longrightarrow \Omega_{X, x}^{p}(\log D)
$$

- изоморфизмы для всех $1 \leqslant p<k_{x}$. Тогда $W_{n}\left(\Omega_{X}^{p}(\log D)\right) \cong \Omega_{X}^{p}(\log D)$ для всех $n \geqslant p$, как и в классическом случае дивизоров с нормальными пересечениями (см. (3.1.8) в [6]). Следовательно, в условиях теоремы 3 определен естественный морфизм $\alpha$ из комплекса $\Omega_{X}^{\bullet}(\log D)$, снабженного фильтрацией Ходжа $F$, в тот же комплекс с убъвающей фильтрацией $W$, заданной формулой $W^{n}=W_{-n}$.

Следствие 5. При тех же предположениях упомянутый выше морфизм $\alpha$ - фильтрованный квазиизоморфизм, если $\mathscr{H}^{i}\left(\omega_{\widetilde{C}^{(n)}}^{\bullet}\right)=0$ nри $i \neq 0$.

Доказательство такое же, как и в (3.1.8.2) работы [loc. cit.]

Замечание 8. Конечно, для дивизоров с нормальными пересечениями вложения (10) - изоморфизмы для всех $p \geqslant 1$. Класс дивизоров, для которых $\sum_{i=1}^{k} \Omega_{X, x}^{1}\left(\log D_{i}\right) \cong \Omega_{X, x}^{1}(\log D)$, рассматривается в теореме (2.9) работы [13] (cp. $\S 2)$.

Замечание 9. Условия из следствия 5 об обращении в нуль когомологий означают, что комплекс регулярных мероморфных форм на нормализации $\widetilde{C}^{(n)}$ ацикличен в положительных размерностях. Наряду со случаем дивизора с нормальными пересечениями, рассмотренного в [6], известно немало типов многообразий, когда это условие выполнено. Среди них - рациональные нормальные 
полные пересечения, факторособенности гладких многообразий с действием конечной группы (см. [9]), торические многообразия, некоторые типы квазиоднородных (строго квазиоднородных) многообразий и др.

Замечание 10. Если оба комплекса $\Omega_{X}^{\bullet}(\log D)$ и $\Omega_{X}^{\bullet}(\star D)$, наделенные стандартной фильтрацией Ходжа, квазиизоморфны (см., например, [8]), то морфизм $\beta$ из предложения (3.1.8) работы [6] - квазиизоморфизм. Если вдобавок выполнено условие предыдущего следствия, то $\alpha$ - также квазиизоморфизм. Это означает, что во всех случаях, перечисленных в предыдущем замечании, существуют изоморфизмы (3.1.8.2) из работы [loc. cit.]:

$$
R^{n} j_{*} \mathbf{C} \cong \mathscr{H}^{n}\left(j_{*} \Omega_{X^{*}}^{*}\right) \cong \mathscr{H}^{n}\left(\Omega_{X}^{\bullet}(\log D)\right),
$$

где $X$ - некоторое многообразие, $X^{*}=X \backslash D, j: X^{*} \rightarrow X-$ каноническое вложение.

Дальнейший анализ показывает, что при одном из стандартных предположений на объемлющее многообразие $X$ (гладкость, кэлеровость, полнота и др.) бифильтрованный комплекс $\left(\Omega_{X}^{\bullet}(\log D), F, W\right)$ можно использовать (см. [14, c. 532]) для вычисления канонической смешанной структуры Ходжа на когомологиях дополнения $H^{*}(X \backslash D, \mathbb{C})$ и на локальных когомологиях $H_{C}^{*}\left(X, \Omega_{X}^{\bullet}(\log D)\right)$ без использования теорем о разрешении особенностей и стандартной редукции к случаю нормальных пересечений.

В заключение отметим, что дифференциал $d$ строго совместим [6, (1.1.5)] в степени $k+1$ с весовой фильтрацией $W$, т. е.

$$
d \Omega_{X}^{k}(\log D) \cap W_{n}\left(\Omega_{X}^{k+1}(\log D)\right)=d\left(W_{n}\left(\Omega_{X}^{k}(\log D)\right), \quad n \in \mathbb{Z} .\right.
$$

Тем самым определена весовая фильтрация на усеченном логарифмическом комплексе де Рама $\tau_{\geqslant k} \Omega_{X}^{\bullet}(\log D)$, которая с помощью отображения кратного вычета индуцирует весовую фильтрацию на комплексе регулярных мероморфных форм полного пересечения, причем в этом случае выполнения условий (10) уже не требуется.

\section{ЛитеРАТУРА}

[1] A. G. Aleksandrov, Non-isolated hypersurface singularities, in: Theory of Singularities and Its Applications, Advances in Soviet Mathematics, vol. 1, Amer. Math. Soc., Providence, RI, 1990, 211-246.

[2] A. G. Aleksandrov, A. K. Tsikh, Théorie des résidus de Leray et formes de Barlet sur une intersection complète singulière, C. R. Acad. Sci. Paris, Ser. I, 333:11 (2001), 973-978.

[3] A. G. Aleksandrov, A. K. Tsikh, Multi-logarithmic differential forms on complete intersections, Ж. Сибирск. федерального ун-та, сер. матем., физ. (изд-во СФУ, Красноярск), 1:2 (2008), 105-124.

[4] D. Barlet, Le faisceau $\omega_{X}^{\bullet}$ sur un espace analytique $X$ de dimension pure, Lecture Notes in Math., vol. 670, Springer-Verlag, Berlin, 1978.

[5] D. A. Buchsbaum, D. S. Rim, A generalized Koszul complex. II. Depth and multiplicity, Trans. Amer. Math. Soc., 111 (1964), 197-224.

[6] П. Делинь, Теория Ходжа II, Математика (сб. пер.), 17:5 (1973), 3-56.

[7] Ф. Гриффитс, Дж. Харрис, Приниипь алгебраической геометрии, т. 2, Мир, М., 1982.

[8] M. P. Holland, D. Mond, Logarithmic differential forms and the cohomology of the complement of a divisor, Math. Scand., 83:2 (1998), 235-254. 
[9] M. Kersken, Reguläre Differentialformen, Manuscripta Math., 46:1 (1984), 1-25.

[10] E. Kunz, Holomorphe Differentialformen auf algebraischen Varietäten mit Singularitäten. I, Manuscripta Math., 15 (1975), 91-108.

[11] K. Morita, On the basis of twisted de Rham cohomology, Hokkaido Math. J., 27:3 (1998), 567-603.

[12] K. Saito, On a generalization of de-Rham lemma, Ann. Inst. Fourier (Grenoble), 26:2 (1976), 165-170.

[13] K. Saito, Theory of logarithmic differential forms and logarithmic vector fields, J. Fac. Sci. Univ. Tokyo, ser. IA, 27:2 (1980), 265-291.

[14] J. H. M. Steenbrink, Mixed Hodge structure on the vanishing cohomology, in: Real and Complex Singularities (Proc. Nordic Summer School, Symp. Math., Oslo, 1976), Sijthoff and Noordhoff Publ., Alphen aan den Rijn, 1977.

[15] H. Wiebe, Über homologische Invarianten lokaler Ringe, Math. Ann., 179:4 (1969), $257-274$.

Институт проблем управления РАН

Поступило в редакцию

e-mail: ag_aleksandrov@mail.ru

28 декабря 2011 г. 